\title{
Giant hemangioma of anterior mediastinum mimicking bilateral pleural effusion
}

\author{
Krystian Pawlak, Cezary Piwkowski, Szymon Smoliński, Wojciech Dyszkiewicz \\ Department of Toracosurgery, Poznan University of Medical Sciences, Poland
}

Kardiochirurgia i Torakochirurgia Polska 2017; 14 (4): 282-284

Hemangioma is a rare mediastinal tumor corresponding to approximately $4-8 \%$ of all benign tumors in children and less than $0.5 \%$ in the overall population [1]. In more than half of cases it is situated in the anterior mediastinum and in $75 \%$ it affects young patients under 35 years of age in similar frequency in both sexes [1, 2].

The case of a huge hemangioma in the anterior mediastinum, radically removed during thoracotomy, diagnosed in a 22-year-old patient, is presented.

A 22-year-old man, without history of disease, was admitted to the hospital in a severe general condition with respiratory failure. The patient reported dyspnea at rest, increasing for 2 months. On the day of admission, all laboratory tests, including $\beta$-human chorionic gonadotropin $(\beta$-HCG) and $\alpha$-fetoprotein (AFP), did not reveal any abnormalities, except for an arterial blood gas test that demonstrated significant hypoxemia. The patient had not been subjected to a chest radiograph before.

The chest radiograph demonstrated a total opacity of the left and 2/3 of the upper half of the chest (Fig. 1). In the chest computed tomography (CT) scan, an encysted extensive nodular lesion, filled with a heterogeneous fluid, was described (Fig. 2). The tumor filled almost the entire left pleural cavity, causing atelectasis of the left lung, and displaced the mediastinum with the heart and large vessels to the right side. The lesion also filled the upper and anterior part of the right pleural cavity, arranged along the anterior chest wall from the diaphragm to the level of the $4^{\text {th }}$ intercostal space, with atelectasis of the middle lobe. Echocardiography did not reveal any impairment of the heart or pericardial cavity. The ultrasound examinations of the abdomen, neck and testicles were normal.

Because of life-threatening symptoms the left pleural cavity was punctured, evacuating approximately $1500 \mathrm{ml}$ of bloody fluid. A cytological examination of the pleural fluid was negative. After improvement in the patient's general condition was achieved, diagnostic left-sided video-assisted thoracic surgery (VATS) was performed. During the procedure, after the introduction of trocars, significant bleeding from the pleural cavity was observed. Video-assisted thoracic surgery was immediately converted to left anterolateral thoracotomy, under the fifth rib, and a cystoid, smoothly encysted elastic tumor involving both pleural cavities and the anterior mediastinum was recognized. The tumor, filled with $6200 \mathrm{ml}$ of bloody fluid, was attached to the chest wall, mediastinum and diaphragm by soft adhesions. The tumor's vessels were derived from the internal thoracic artery and brachiocephalic vein. Finally, the tumor underwent radical resection (Fig. 3).

In histological examination an arteriovenous hemangioma was identified. The tumor had the dimensions $25 \times 14$ $\times 5 \mathrm{~cm}$ and consisted of numerous cystic spaces containing dark cherry-colored fluid. The diagnosis was confirmed by immunohistochemistry based on positive results of reactions of CD31, CD34, SM A markers and desmin.

Because of anemization, in the perioperative period the patient required a transfusion of four blood units. Otherwise, there were no complications in the early postoperative period. Chest tubes were removed on postoperative day 5 . The control chest radiograph demonstrated a normal lung re-expansion (Fig. 3). The patient was discharged home in good general condition on postoperative day 13. Currently, the patient, without clinical symptoms, is undergoing routine outpatient follow-up. The chest radiograph performed six months ago did not demonstrate any abnormalities.

Mediastinal hemangiomas are usually well defined and encysted, and tumor size can range from 2 to over $20 \mathrm{~cm}$ [3]. Histologically, hemangiomas are divided into cavernous, capillary hemangiomas, which correspond to approximately $90 \%$ of all mediastinal hemangiomas, and rare venous and arteriovenous hemangiomas. In half of cases, the disease is asymptomatic and is diagnosed accidentally during a performed chest radiograph; however, in some patients such nonspecific symptoms as cough, dyspnea or chest pain and shortness of breath may occur. In the case of compression or infiltration of the surrounding structures, dysphagia, superior vena cava syndrome (SVCS), Horner's syndrome, or neurological symptoms may develop $[1,4]$.

Although in the literature mediastinal hemangiomas of a significant size, measuring up to $20 \mathrm{~cm}$, are described,

Address for correspondence: Krystian Pawlak MD, Department of Toracosurgery, Poznan University of Medical Sciences, 62 Szamarzewskiego St, 60-569 Poznań, Poland, phone: +48 509835 550, e-mail: krystianp@hotmail.com

Received: 26.02.2017, accepted: 15.06.2017. 


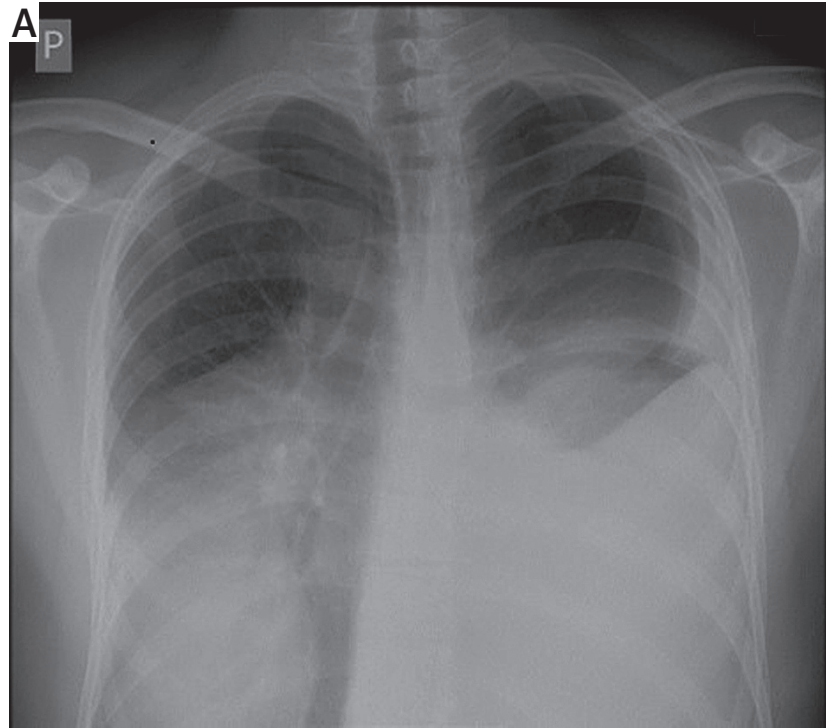

Fig. 1. Chest X-ray at admission to the hospital

the authors have not found a report concerning such a large vascular lesion, covering both pleural cavities, to date. In radiological examinations, including chest $\mathrm{CT}$, the tumor was mimicking a pleural effusion. Similar cases were described by Kubokura et al. and Deepak et al. [4, 5]. In chest CT scan no phleboliths or calcifications characteristic for hemangiomas were observed [2]. Due to the lack of characteristic radiographic features and normal indicators of laboratory blood tests, preoperative diagnosis of the tumor is very difficult. Performing a biopsy in large tumors appears to be ineffective, and moreover is associated with a higher risk of complications, primarily bleeding [1, 4].

In the case of most mediastinal hemangiomas, a radical resection is the treatment of choice, although it is associated with a significant risk of blood loss. This is a result of increased bleeding due to adhesions of the tumor with

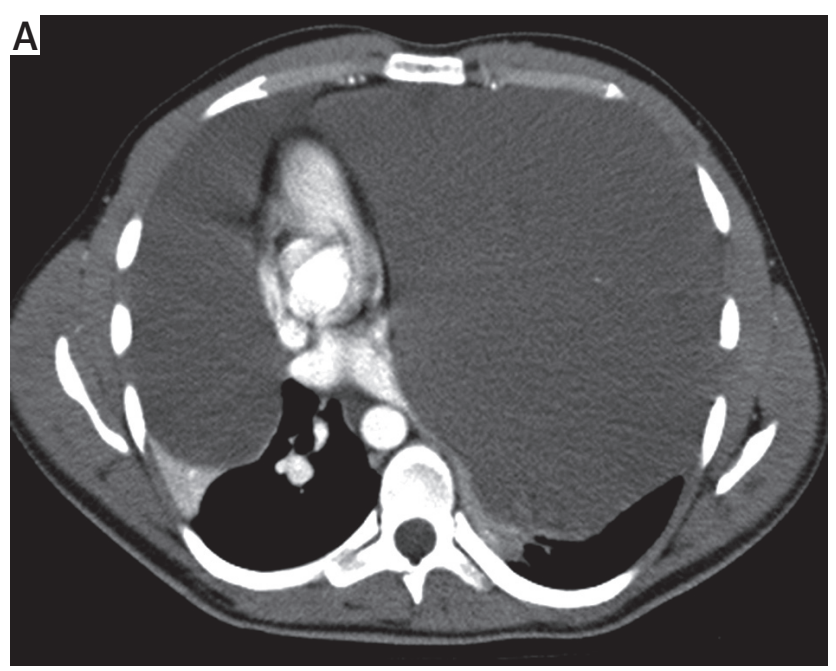

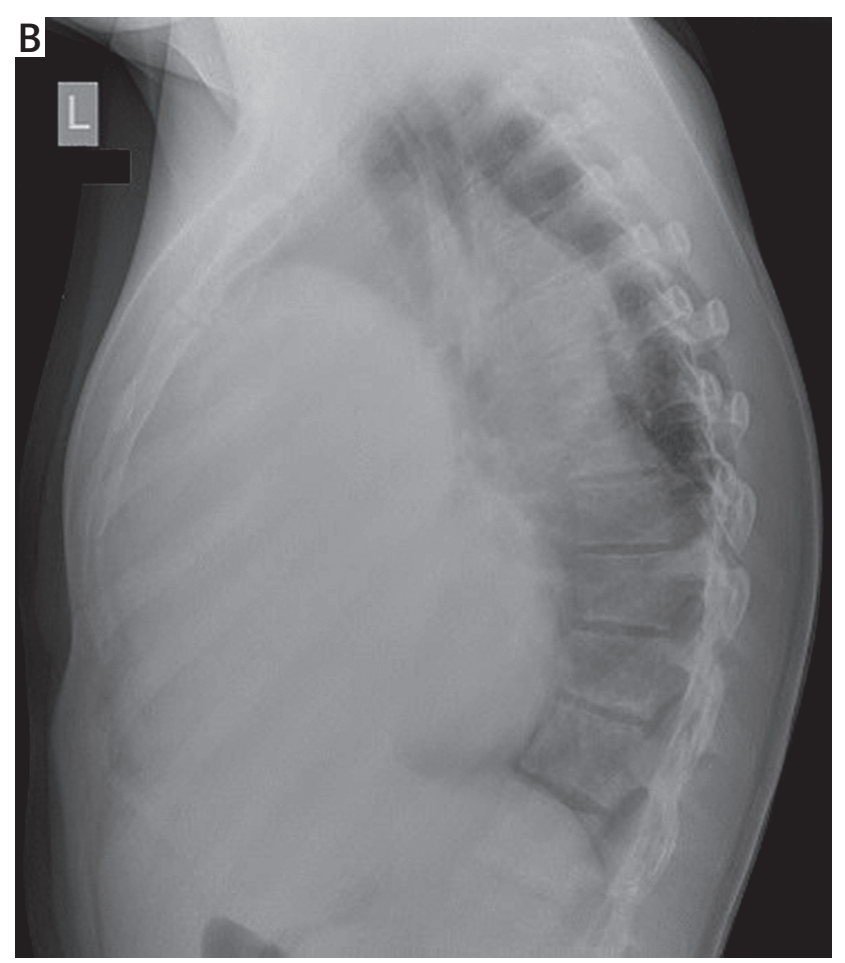

the surrounding structures [4]. Selection of a correct surgical approach is essential to achieve radicality and safety of the procedure. Video-assisted thoracic surgery is justified in the case of small, limited vascular lesions [4, 5]. However, in the case of large, especially bilateral tumors, the correct surgical approach should be obtained by sternotomy. Radical resection by left thoracotomy described in this case was possible; however, it resulted from the late intraoperative diagnosis of the tumor and complications during diagnostic VATS. In the authors' opinion, in the case of intraoperative bleeding conversion to thoracotomy was more justified than sternotomy.

Incomplete resection is associated with a high risk of recurrence of a neoplasm, although Cohen et al. observed 6 patients subjected to non-radical hemangioma resections, who did not relapse in the long-term postoperative period [1].

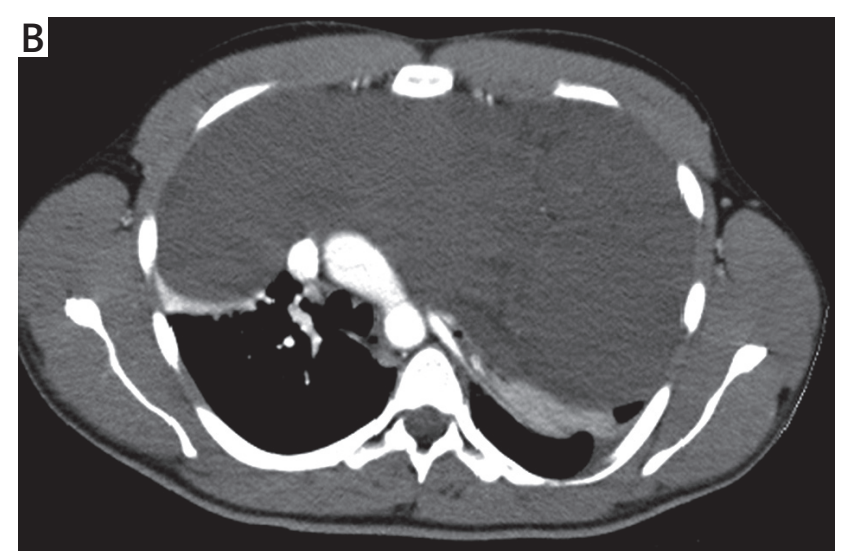

Fig. 2. Preoperative chest computed tomography 

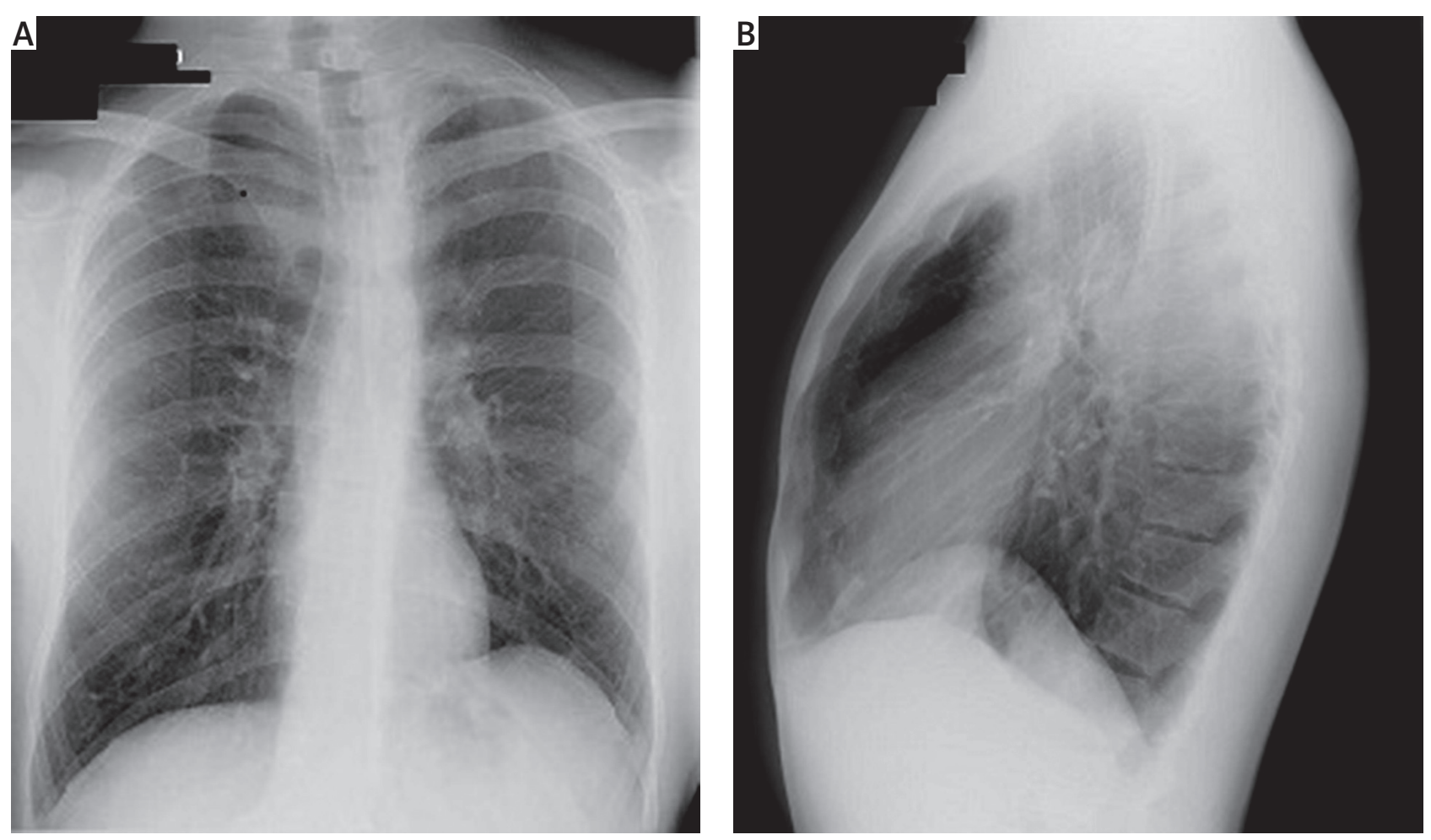

Fig. 3. Chest X-ray performed 6 months after surgery

In cases when radical surgical resection is difficult, incomplete resection may be helpful in order to reduce the tumor mass and decrease the pressure of the tumor on the surrounding organs and reduce the risk of hemorrhage $[4,5]$.

Mediastinal hemangiomas are extremely benign tumors; however, in radiological examination they can imitate massive effusion in the pleural cavity. This should be considered in the case of a spontaneously occurring effusion, especially in young patients.

\section{Disclosure}

Authors report no conflict of interest.

\section{References}

1. Cohen AJ, Sbaschnig RJ, Hochholzer L, Lough FC, Albus RA. Mediastinal hemangiomas. Ann Thorac Surg 1987; 43: 656-659.

2. Davis JM, Mark GJ, Greene R. Benign blood vascular tumors of the mediastinum. Report of four cases and review of the literature. Radiology 1978; 126: 581-587.

3. Moran CA, Suster S. Mediastinal hemangiomas: a study of 18 cases with emphasis on the spectrum of morphological features. Hum Pathol 1995; 26: 416-421.

4. Kubokura H, Okamoto J, Hoshina H, Ishii H, Koizumi K, Shimizu K. Mediastinal cystic hemangioma presenting as bilateral bloody pleural effusion: a case report. J Nippon Med Sch 2012; 79: 381-347.

5. Deepak J, Narendra Babu M, Gowrishankar B, Ramesh S. Mediastinal hemangioma: masquerading as pleural effusion. J Indian Assoc Pediatr Surg 2013; 18: $162-164$. 